\title{
Construction de soi à travers un dispositif de carnets littéraires
}

\section{Carol Battistini}

\section{(2) OpenEdition}

1 Journals

Édition électronique

URL : http://journals.openedition.org/trema/2537

DOI : 10.4000/trema.2537

ISSN : 2107-0997

Éditeur

Faculté d'Éducation de l'université de Montpellier

\section{Édition imprimée}

Date de publication : 1 décembre 2010

Pagination : $54-63$

ISSN : 1167-315X

\section{Référence électronique}

Carol Battistini, « Construction de soi à travers un dispositif de carnets littéraires », Tréma [En ligne], 33 - 34 | 2010, mis en ligne le 01 décembre 2012, consulté le 19 avril 2019. URL : http:// journals.openedition.org/trema/2537 ; DOI : 10.4000/trema.2537

Ce document a été généré automatiquement le 19 avril 2019

Trema 
Construction de soi à travers un dispositif de carnets littéraires

Carol Battistini

I. Contexte de recherche 
1 La construction de soi est ici envisagée comme un processus interactif entre un sujet et le milieu socioculturel dans lequel il est immergé. Elle est liée au sentiment et à la possibilité de constituer un soi organisé dans un contexte donné et reposant sur une réelle estime de soi-même (Erikson, 1972). Cette recherche-action dans l'objectif qu'elle s'est fixé : permettre un agrandissement de soi à travers la relation à la littérature, se propose de mettre en synergie un être de désir au sens spinoziste du terme, c'est-à-dire habité par une puissance de jouir et d'agir (Spinoza,1954) et une classe de littérature comme réalité sociale pourvoyeuse de repères identitaires (communauté de lecteurs et d'auteurs, oeuvres littéraires), d'outils structurants (apprentissages

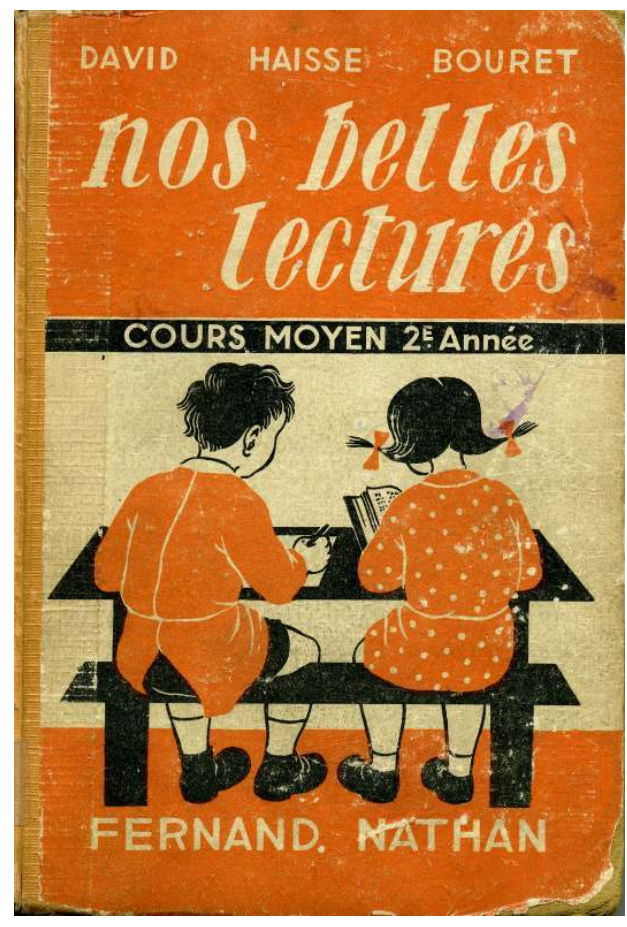
littéraires) et d'un cadre facilitateur

(présence et mise en espace des livres dans la classe, existence de moments disponibles pour lire et écrire).

Cette réflexion sur le désir de littérature à travers l'écriture et la lecture intègre également la notion de travail créateur en tant que processus marqué par différentes étapes : saisissement créateur, notation des matériaux refoulés, institution d'un code, composition, socialisation de l'œuvre (ANZIEU, 1981) et comme inscription de soi au monde. Elle s'appuie aussi sur la vision barthienne du plaisir du texte, espace de rencontre entre un auteur et un lecteur, lieu où s'éprouve le désir de l'autre (BARTHES, 1973).

Dans le champ de la didactique du français, la recherche se réfère à différentes conceptions de l'écriture parfois élaborées aux marges du système éducatif : écriture comme libre expression de soi (FREINET, 1969, BING, 1976), comme fruit d'un transfert dans une dynamique lecture/écriture (TAUVERON \& Sève, 2005), comme processus créateur (ANDRE, 1987). Elle s'appuie également sur les travaux de BUCHETON montrant l'importance d'un espace social de partage et d'échange pour affirmer et construire une identité de sujet écrivant (BUCHETON, 2002).

\section{Le dispositif des carnets littéraires : description, enjeux, signification}

2 On met à disposition de l'élève un espace personnel où il peut recueillir des textes qu'il apprécie et qu'il s'approprie activement en les inscrivant dans le lieu de ses préférences. Dans ce carnet, l'élève peut aussi essayer sa propre écriture et se dire en toute subjectivité. Ces textes peuvent être exposés lors de rencontres bimensuelles.

Le dispositif des carnets littéraires est mené parallèlement à des apprentissages littéraires dirigés : ateliers poétiques à dominante linguistique et imaginaire (JOLIBERT, 1998), connaissance et exploration de genres et de procédés littéraires (TAUVERON \& SEVE, 2005) mettant en lien lecture et écriture, procédures de révision de texte (EVA, 1991). 
Cependant si le désir de littérature peut se construire à travers la relation didactique, son émergence émane d'un sujet qui requiert pour s'affirmer des espaces de liberté inaliénables. Aussi la nécessaire "instrumentation» de l'élève, doit-elle s'accompagner d'une activité vécue comme une libération de l'acte d'écrire.

Bien qu'émanant de la sphère intime, l'écriture, pour se déployer, appelle une sociabilité stimulante car l'implication de l'élève est en lien avec la réception qu'il imagine pour ses textes (BUCHETON \& CHABANNE, 2002). Pour devenir amateur/auteur, l'élève doit s'intégrer à une communauté de pairs où il peut partager le plaisir de ses découvertes à travers une lecture à haute voix pour éveiller chez d'autres, les émotions qu'il a lui-même éprouvées. Lisant ses propres productions, il peut apprendre à assumer une parole d'auteur et à accepter le regard d'autrui sur ses» inventions littéraires ». Enfin la présence d'un public est une incitation à travailler l'écrit et à inscrire le lecteur/auditeur dans le texte.

\section{Méthodologie retenue}

\section{1. Axes d'investigation}

3 Se lancer dans l'aventure des carnets littéraires est en soi l'expression d'un désir de littérature s'accompagnant d'une construction de soi dont les traces sont ici mises en relief. Réflexion théorique et observation des matériaux recueillis ont permis de construire trois axes d'investigation qui ne sont pas des catégories étanches mais plutôt des « orientations dynamiques » de la recherche.

\section{1. 1. L'appropriation du texte : saisi, incorporé, digéré}

4 Le premier type d'investigation porte sur les actes d'appropriation de l'amateur de textes et concerne les écrits strictement recopiés et "adoptés ». Avant d'inscrire le texte sur le carnet, comment l'élève s'en saisit-il : se met-il en état de recherche/d'affût ou de réception active? La présence de cet écrit témoigne d'une intention, d'une attention, d'une énergie tournée vers la « littérature ». Par la suite, quel est le devenir du texte ? Au-delà du compagnonnage dans l'espace intime du carnet, le destine-t-on à être partagé avec des pairs ? Est-ce un point d'appui à une production ultérieure?

Le second type d'investigation porte sur les actes d'appropriation de "l'auteur/amateur " de littérature se manifestant par la présence de textes d'emprunt intégrés aux textes produits. Cette présence est plus ou moins forte : l'apport du jeune auteur qui s'essaye peut être de l'ordre de la retouche, il peut aussi utiliser un texte matrice autour duquel bâtir son propre texte, ou ne garder en filigrane qu'un thème ou une idée du texte d'origine.

\section{1. 2. Recherche d'une relation euphorique avec le lecteur ou l'auditeur du texte : se construire à travers la relation à l'autre}

5 Le désir de tisser une relation euphorique avec le lecteur/auditeur peut s'exprimer chez l'amateur de textes et bien sûr chez l'auteur apprenti qui tente d'établir une relation artistique avec son public, c'est-à-dire d'être reconnu dans ses intentions. Cet axe porte sur tout type d'écrit, bien que l'enjeu identitaire ne soit pas le même que l'on soit auteur ou amateur de textes. 
Le texte est-il un appel au lecteur/auditeur potentiel dont l'auteur ou l'amateur recherche les réactions et la complicité ? Du côté de l'auteur : on peut faire l'hypothèse que l'écriture fait pendant à la lecture et que l'élève ayant expérimenté la lecture littéraire comme un jeu à partir d'un texte qui $a$ du jeu et le sens $d u$ jeu, doit pouvoir à son tour construire pour le lecteur un tel type de texte (TAUVERON, 2002). Les élèves adoptent-ils une posture d'auteur recherchant un partenaire investi et réactif? Du côté de l'amateur : le choix du texte et sa présentation n'ont-ils pas pour objectif de provoquer chez l'auditeur, des explorations ludiques, des émotions ou des questions suscitées lors de sa propre lecture?

Le texte est-il adressé ? C'est-à-dire choisi ou conçu en imaginant sa réception par son destinataire. Cette adresse témoigne du plaisir d'établir des liens grâce à la littérature (grande ou petite). Le principe même des réunions peut inciter l'auteur à adresser ses textes à un individu mais aussi au cercle d'auditeurs constitué par la classe, alors témoin de la dédicace ou de l'interpellation.

Ces rencontres mettant en présence intention et réception, quels sont les effets de la réception en direct du texte : accueil de la critique des pairs, plaisir du partage complice, test des effets du texte par la lecture à haute voix ? La présence d'un public permet-elle de s'affirmer et de se sentir reconnu comme auteur ou amateur éclairé ?

\section{1. 3. Le travail créateur comme inscription de soi au monde}

6 Cette étude située à l'école élémentaire se propose d'adapter les étapes d'un "travail créateur " au demeurant bien modeste en raison de l'âge des enfants. Cet axe qui étudie les textes inventés ne prend pas en compte les écrits copiés ou retouchés.

La phase préparatoire à la création, le saisissement créateur, réside dans une acuité au monde doublée d'une forte présence à soi-même : tout peut alors faire sens et résonner à l'intérieur de soi. Les élèves témoignent-ils de cet état d'accueil, où le monde, dénaturalisé fait l'objet d'une appropriation/intériorisation, où images, émotions, sensations deviennent des sources d'inspiration?

L'ouverture du chantier d'écriture s'accompagne d'une créativité jaillissante pouvant se manifester par l'exploration de la polysémie, par la recherche d'effets sonores, prosodiques ou graphiques, par les ruptures volontaires avec les normes linguistiques etc. Ce stade présente quelques similitudes avec "la notation des matériaux refoulés" d'ANZIEU, en tant qu'écriture des premiers jets, potentialité demandant à être approfondie et développée pour engager un travail créateur. Cela apparait-il dans les carnets?

L'étape ultérieure est celle du texte travaillé, objet d'opérations multiples et successives qui dénotent une volonté de persévérer dans une entreprise pour réaliser un «objet littéraire ». Préoccupation d'ordre esthétique, motivation à traduire une pensée dans toute sa subtilité, attention portée à la cohérence du texte, positionnement métalittéraire témoignent d'une intention artistique. Celle-ci peut se manifester matériellement au fil des phrases : ratures, flèches, corrections; elle ressort également à l'analyse du texte quand la volonté de maintenir un parti pris apparaît clairement. Cette phase du travail où se mettent en place des stratégies d'écriture plus élaborées serait à mettre en lien avec ce qu'ANZIEU nomme l'institution d'un code et la composition de l'œuvre. Est-il investi par les élèves? 


\section{2. Terrain de recherche}

7 L'étude est mise en place en classe de CM1-CM2 dans une école accueillant des enfants de milieux sociaux contrastés et de niveau scolaire très hétérogène. La gestion du cours double dégage naturellement des espaces de liberté pour les élèves, durant lesquels ils peuvent choisir de lire ou d'écrire. Quatre élèves de la classe ont fait l'objet d'une approche longitudinale. Ils ont comme caractéristiques communes le volontariat, l'âge (10 ans) et leur intérêt pour la lecture mais se différencient par la personnalité, le vécu, le rapport à la norme et le niveau scolaire (satisfaisant pour Luc et Éva, très moyen pour Zoé et Léa).

\section{3. Recueil des données}

8 L'expérimentation du dispositif s'est déroulée sur quatre mois à partir de mars. La recherche a porté sur la totalité des carnets. Des entretiens individuels semi - directifs réalisés fin juin, au fil des cahiers, ont permis de préciser le statut et la provenance des textes, d'obtenir des informations sur la dynamique d'écriture et les intentions d'auteur des élèves, et enfin de connaître les effets des rencontres autour des carnets. Par ailleurs, le calendrier des "lectures offertes" et le cahier journal de l'enseignante ont mis à disposition de précieuses informations sur l'apport didactique et culturel dont les élèves ont bénéficié en amont de la démarche. Certaines lectures personnelles mentionnées par les élèves ont fait l'objet d'études comparatives. Une lecture flottante des matériaux recueillis a tout d'abord permis de faire ressortir les trois axes d'investigation présentés qui ont ensuite été traités plus finement à l'aide de grilles d'analyse. Données suscitées, provoquées et invoquées (VAN DER MAREN, 2003) ont ensuite été rassemblées et mises en lien autour de chaque analyseur comme dans l'exemple ci-dessous :

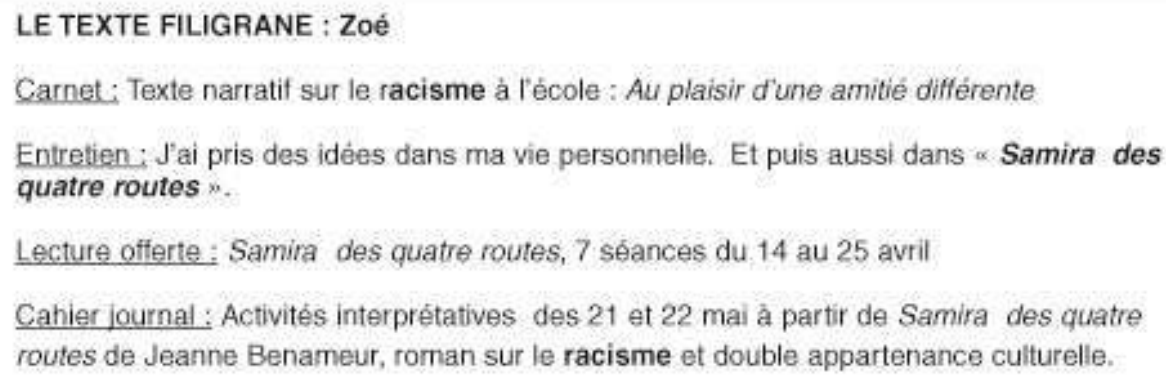

\section{Un bref aperçu des résultats}

9 Afin de présenter de manière très succincte cette étude, nous nous appuierons sur la totalité des données mais ne présenterons que quelques exemples significatifs pour illustrer nos propos.

Globalement la dimension d'appropriation est centrale pour les quatre cas étudiés et concerne cinquante-deux textes sur les soixante et onze du corpus.

Les quatorze écrits copiés par Éva, Luc et Léa sont des textes courts et ludiques : blagues, devinettes, charades. Les élèves les ont activement recherchés dans des recueils, des 
bandes dessinées ou des supports plus insolites comme les yaourts de la cantine mentionnés par Éva. Ces textes ont aussi pu être racontés, retenus et transcrits. Seul Luc témoigne d'un projet pour ces textes importés : ses blagues sont clairement destinées à être partagées avec ses pairs («Pour les blagues, je pense que mes copains vont rigoler») et une charade recopiée, composée de trois définitions identiques, donne lieu à la création d'une nouvelle charade construite sur le même principe : "Y'en a une qu'on m'a donnée et l'autre que j'ai inventée ». Les deux écrits se côtoient d'ailleurs sur la page.

Trente-huit textes, répartis dans les quatre carnets, attestent de la présence d'autres écrits. La moitié des emprunts provient des apprentissages littéraires. Seule Léa utilise aussi des lectures personnelles. Elle s'approprie les textes du Fil à retordre de BOURGEYX en les retouchant. Cette opération peut consister à actualiser la monnaie : «Ce qui est de moi, c'est ce qui est traduit en euros " (Léa à propos de La machine à gondoler), ou à pousser à l'extrême l'absurdité d'une situation : "L'idée du livre, c'est que tout le monde allait être obligé de croire au Père Noël. Je me suis dit que pendant qu'ils z' y étaient, ils pouvaient même mettre des détecteurs de mensonges, comme ça, y'avait plus qu'une chose à faire, c'était de rester chez soi " (Léa à propos de Père Nono). Les quatre écoliers utilisent des textes matrices, principalement des textes poétiques en provenance de la classe. Ainsi Éva et Luc ont-ils décliné «Une fourmi de dix-huit mètres» de Desnos: ils en ont repéré et conservé l'ossature linguistique et se sont lancés dans la description surréaliste d'autres animaux. Le texte filigrane est le seul fait de Zoé : derrière une production personnelle intitulée $\mathrm{Au}$ plaisir d'une amitié différente se profile un roman de Jeanne BENAMEUR étudié en classe et qui, comme le récit de Zoé, parle du racisme ordinaire : «J’ai pris les idées dans ma vie personnelle et puis aussi dans « Samira des quatre routes » ».

11 La recherche d'une relation euphorique avec un public apparait dans la totalité du corpus qui présente un large éventail de procédés pour « draguer le lecteur » (BARTHES, 1973).

L'appel au lecteur potentiel se manifeste de différentes manières. Tout d'abord, à travers l'intention de surprendre en créant des situations inédites, ce que tente de faire Léa à travers un texte intitulé Déclaration d'amour à mon cartable et qu'elle commente ainsi : «Ça change des lettres d'amour habituelles!». Il s'agit aussi d'établir des connivences autour de «l'univers facétieux de l'enfance ». Ainsi Zoé, dans Homo sapiens ignoriste, pour mettre en scène un humain régressif, déconstruit le langage, redouble de propos scatologiques et prend les adultes pour cibles, en particulier une maitresse qui ne sait plus parler et risque d'être "euthanasiée ». Dans une tout autre optique, elle interpelle son public sur ses valeurs : Au plaisir d'une amitié différente, invite à réfléchir à l'amitié possible au-delà des différences culturelles, car finalement ainsi qu'elle conclut son récit: «tout le monde est pareil».

La pratique du texte adressé est courante chez Luc, Zoé et Léa, elle traverse tous les types de textes (narratifs, descriptifs et ludiques), concerne souvent les pairs, parfois aussi les enseignants. Ainsi Léa brosse trois portraits humoristiques de son maitre (« Alors je lui ai refait une description en m'amusant bien ») et les lui adresse publiquement lors des réunions. Tous les élèves témoignent des effets de la réception en direct: ils sont sensibles au regard du groupe sur leurs productions et testent les effets de leurs textes. Interrogée sur l'intérêt des réunions, Éva témoigne: «Pour moi, c'est important. Tu peux savoir ce que les autres pensent de ce que tu écris ». Zoé illustre bien la jubilation de l'auteur/interprète dont l'attente est comblée par les réactions du public: "Dans le poème que je leur ai lu, j'avais écrit : « Mais le pire, c'est sous mon drap, d'avoir un rat, logé avec sa nana », y z'ont rigolé et j'étais trop contente! ». En tant qu'auteur, elle seule exprime clairement qu'elle se sent valorisée 
et satisfaite d'elle-même : «J'aime bien lire mes histoires, je suis contente de moi ».

Le travail créateur, bien qu'inégal, apparaît par touches chez les quatre écoliers.

La phase préparatoire à l'écriture qui se traduit par une forte présence au monde, à soimême, à la littérature, est particulièrement investie par Léa et Zoé. Cette dernière, attentive à son environnement, met en relief des principes qu'elle transpose dans une "création littéraire». Ainsi le fil conducteur d'Homo sapiens ignoriste provient de l'observation de la frise chronologique de la classe associée au principe de l'écriture arabe évoquée dans un roman lu en classe : "J'ai regardé devant moi la frise historique, on la lit de gauche à droite, ça commence par la préhistoire, c'est l'homo sapiens sapiens, il invente le feu et petit à petit y vit mieux. J'ai voulu inventer un nouvel être: " homo sapiens ignoriste " c'est le contraire de sapiens sapiens. Lui, y devient de plus en plus bête, il retourne à la préhistoire, il sait même plus parler». Inventant une évolution "de droite à gauche! Comme l'écriture des Arabes!», elle organise un récit autour de l'idée d'une évolution régressive de l'être humain.

La créativité jaillissante est le fait de tous les élèves et se manifeste par la création de nouveaux langages, la recherche d'effets sonores et le travail sur la polysémie. Ainsi Éva, s'essayant au mot-valise, montre qu'elle en maîtrise la construction, proposant deux mots qui s'accolent et qui font image : "marinfirmière : c'est un marin qui joue à l'infirmière »; elle ne parvient cependant pas à faire aboutir la phase plus délicate de la définition consistant à jouer sur les sens des deux mots et sur celui qu'ils prennent quand ils sont soudés, sans jamais nommer littéralement l'une ou l'autre des composantes. L'intention de jouer sur le sens des mots existe mais se situe au stade de l'exploration.

Le travail sur le texte apparaît dans les quatre carnets, mais n'est longuement explicité que par Zoé. Celle-ci témoigne d'un réel retour sur ses écrits au-delà de l'aspect jaillissant des premières intuitions littéraires : ainsi dans La fille au plaisir d'une amitié différente son souci d'assurer la progression textuelle se manifeste par les traces de corrections apportées au texte (ratures, flèches, utilisation du «blanco »), ce qu'elle commente lors de l'entretien: "Au début, [...] j'ai voulu dire: «On se moquait de Kaïma et puis...ça la dégoûtait " et puis après j'avais enchaîné exactement » Elles étaient meilleures amies ", mais j'avais pas encore parlé de Camille, alors il fallait que je parle de Camille aussi ; alors j'ai tout barré et puis j'ai récrit. Pour Homo sapiens ignoriste, ses hésitations pour choisir un titre, montrent à la fois sa préoccupation de traduire le plus justement sa pensée mais aussi d'être cohérente avec le récit qu'elle a produit: " "Vulgariste », ça faisait trop "vulgaire », alors " ignoriste " plutôt, parce que c'est des gens ...bêtes! [...], j'ai choisi "ignoriste " parce qu'elle ne sait pas parler». L'adoption d'un positionnement métalittéraire apparaît à plusieurs reprises chez Léa ; celle-ci, par exemple, conclut son carnet par un texte qu'elle intitule La pleurnicharde et qu'elle commente ainsi : " comme on était en fin de carnet littéraire, j'ai fait un petit qui pleurait à sa mère que ... le carnet littéraire, il était fini. » Ainsi à travers un dernier écrit, Léa met en scène la tristesse du cahier qui s'achève.

Globalement, deux profils semblent se dégager parmi ces élèves.

Éva et Luc, « bons » élèves respectueux de la norme scolaire, se sont largement approprié des textes, recopiant mais aussi prolongeant, déclinant des écrits rencontrés en classe : cela témoigne de leur capacité d'accueil puis de réinvestissement personnel des activités scolaires. La créativité de ces deux élèves s'exerce quand ils disposent d'un cadre formel sur lequel s'appuyer. On peut dire et sans porter de jugement aucun, qu'ils se situent plutôt dans un contrat scolaire.

A l'opposé, Zoé et Léa, élèves moins conformes aux attentes scolaires, utilisent des sources d'inspiration multiples, tentent des explorations linguistiques et littéraires et 
donnent à voir un réel travail sur le texte (même maladroit ou inabouti). Elles osent un rapport plus personnel à la littérature, puisant dans leurs lectures privées (Léa) ou faisant un usage original des textes rencontrés en classe (Zoé). Toutes deux sont réceptives au monde environnant et l'utilisent pour inventer : il alimente leur créativité jaillissante ou fournit un code organisateur à leurs écrits. L'examen des textes produits donne une impression de foisonnement créatif et de libération de la parole. Léa et Zoé vont au-delà du contrat scolaire et s'emparent de l'écriture littéraire comme expression et affirmation de soi.

Cette étude, portant sur un nombre restreint de cas, ne permet pas de tirer des conclusions d'ordre général. Toutefois, comment ne pas mettre en lien ces deux profils d'élèves avec la thèse de KAÏCI montrant à travers l'analyse de deux cents rédactions que les bons élèves sont ceux qui perçoivent le mieux la norme scolaire et s'y conforment, produisant des textes plus stéréotypés, alors que des élèves "moins performants" repèrent mal les attentes de l'institution mais produisent des textes plus investis et plus originaux (KAÏCI, 1991). Cette spontanéité et cette indocilité sont bien le fait de Léa et de Zoé qui trouvent à se déployer dans un espace de liberté où la prise de risque n'est jamais scolairement sanctionnée. On remarque ici que cette créativité loin de s'opposer aux apprentissages littéraires plus formels (et utiles, l'intégration d'une norme étant nécessaire à sa transgression), s'en nourrit mais d'une manière toujours singulière et parfois surprenante. Le dispositif des « carnets littéraires " s'inscrit donc dans un projet de faire de l'écriture une aventure personnelle, prenant en compte le sujet écrivant dans ces composantes affectives et culturelles (SORIN, 2005). En ce sens, il est ouvert à tous les enfants, en particulier à ceux qui ne sont pas particulièrement valorisés par l'école, à condition bien sûr que l'enseignant quitte un temps la posture de correcteur pour adopter celle d'un lecteur/auditeur attentif et curieux des intentions de ses élèves.

\section{BIBLIOGRAPHIE}

\section{OUVRAGES THÉORIQUES}

ANDRÉ, A. (1987). Babel Heureuse. Paris : Syros.

ANZIEU, D. (1981). Le corps de l'œuvre. Paris : Gallimard

BARTHES, R. (1973). Le plaisir du texte. Paris : Editions du Seuil.

BATTISTINI, C. (2008). Désir de littérature et pratiques didactiques, Thèse de doctorat, Université de Toulouse 2, Département des Sciences de l'Education.

BING, E. (1976). Et je nageai jusqu'à la page. Paris : des femmes

BUCHETON, D. \& CHABANNE, J.C. (2002). Parler et écrire pour penser et se construire. L'écrit et l'oral réflexifs. Paris : PUF

BUCHETON, D. (2002). «Devenir l'auteur de sa parole». Prendre la parole. Apprendre la parole. Apprendre par la parole, Saint Nazaire, du 12 au 27 juillet 2002. 
Actes du colloque [en ligne] $h t t p: / / w w w . e d u s c o l . e d u c a t i o n . f r / D 0033 /$ parole_bucheton.pdf

ERIKSON, E. (1972). Adolescence et crise. La quête de l'identité. Paris : Flammarion

FREINET, C. (1969). Pour l'école du peuple. Paris : Petite collection Maspero

JOLIBERT, J. (1992). Former des enfants lecteurs et producteurs de poèmes. Paris : Hachette Education.

KAї̈I, A. (1991). La pratique de la rédaction au cycle d'observation des collèges. Analyse des effets de

quelques modes de sollicitation à l'écriture des élèves en échec. Thèse de doctorat. Université de

Bordeaux 2.

SORIN, N. (2005). » Vers une didactique de l'écriture littéraire du récit de fiction au primaire », Nouveaux

cahiers de la recherche en éducation, vol $8, \mathrm{n}^{\circ} 1,65-78$.

SPINOZA B. (1954). L'Éthique. Paris : Gallimard.

TAUVERON, C., \& Sève, P. (2005). Vers une écriture littéraire. Paris : Hatier.

VAN DER MAREN, J.M. (2003). La recherche appliquée en pédagogie. Bruxelles : De Boeck.

LITTÉRATURE DE JEUNESSE

BENAMEUR, J. (1992). Samira des quatre routes. Paris : Flammarion

BOURGEYX, C. (1991). Le fil à retordre. Paris : Nathan

DESNOS, R. (1970). Chantefables et chantefleurs. Paris : Gründ

\section{RÉSUMÉS}

La classe de littérature peut-elle favoriser la construction identitaire des élèves ? Telle est la question à laquelle tente de répondre cette contribution à travers les résultats d'une rechercheaction ayant pour objectif de faire croître le désir de littérature d'élèves de cours moyen. L'étude se focalise sur un dispositif didactique nommé " carnets littéraires », offrant aux élèves un espace personnel pour créer des textes ou recueillir des écrits choisis pouvant être partagés lors de rencontres bimensuelles. Le désir de littérature y apparaît nettement dans sa composante identitaire : l'étude met en relief les actes d'appropriation de textes littéraires tout au long des carnets, la recherche d'une relation euphorique avec le lecteur et l'ébauche d'un travail créateur. Elle montre aussi, comment, à travers un tel dispositif, certains élèves peu conformes à la norme scolaire osent un rapport singulier à la lecture et à l'écriture, se construisant « lecteurs/amateurs de littérature » et « auteurs apprentis ».

Can the literature class support the students' identity development? This is the question this contribution tries to answer through the results of a research-action whereof the objective was to increase the desire in literature of students during the third degree of primary school. The study is focussed on a didactic system called "literary books", it offers to the students a personal space to create texts or to collect chosen writings that can be shared during biweekly meetings. The desire in literature clearly appears in its identity component: the study foregrounds the appropriation acts of literary texts throughout the books, the search of a euphoric connexion with the reader and the draft of a creator work. It also shows, with such a system, how some students who not fit well in the standard education dare an uncommon connexion to the reading and to the writing, building themselves " readers/amateurs in literature " and "trainee authors ». 
INDEX

Mots-clés : appropriation du texte, construction identitaire, désir de littérature, lecteur, relation euphorique, travail créateur

\section{AUTEUR}

CAROL BATTISTINI

Université Toulouse II 